\title{
Medical Pattern Recognition: Applying an Improved Intuitionistic Fuzzy Cross-Entropy Approach
}

\author{
Kuo-Chen Hung \\ Department of Logistics Management, National Defense University, No. 70, Section 2, Jhongyang N. Road, \\ Beitou District, Taipei City 112, Taiwan \\ Correspondence should be addressed to Kuo-Chen Hung, kuochen.hung@msa.hinet.net
}

Received 14 June 2011; Accepted 22 August 2011

Academic Editor: Maysam Abbod

Copyright ( $) 2012$ Kuo-Chen Hung. This is an open access article distributed under the Creative Commons Attribution License, which permits unrestricted use, distribution, and reproduction in any medium, provided the original work is properly cited.

One of the toughest challenges in medical diagnosis is the handling of uncertainty. Since medical diagnosis with respect to the symptoms uncertain, they will be assumed to have an intuitive nature. Thus, to obtain the uncertain optimism degree of the doctor, fuzzy linguistic quantifiers will be used. The aim of this article is to provide an improved nonprobabilistic entropy approach to support doctors examining the work of the preliminary diagnosing. The proposed entropy measure is based on intuitionistic fuzzy sets, extrainformation regarding hesitation degree, and an intuitive and mathematical connection between the notions of entropy in terms of fuzziness and intuitionism has been revealed. An illustrative example for medical pattern recognition demonstrates the usefulness of this study. Furthermore, in order to make computing and ranking results easier and to increase the recruiting productivity, a computer-based interface system has been developed to support doctors in making more efficient judgments.

\section{Introduction}

Medical diagnostic investigations are very important and complex. Uncertainty is an inseparable aspect of medical diagnosis problems. A symptom is an uncertain indication of a disease as it may or may not occur with or as a result of the disease. Uncertainty characterizes a relation between symptoms and diseases $[1,2]$. Hence, coping efficiently with uncertainty leads us to more accurate decision making, and this is considered as a fundamental challenge in medicine.

The fuzzy set framework has been utilized in several different approaches to model the diagnostic process. In the approach formulated by Sanchez [3] in 1979, he adopted the compositional rule of inference by Zadeh [4] as an inference mechanism. It accepts fuzzy descriptions of a patient's symptoms and infers fuzzy descriptions of patient's diseases by means of the fuzzy relationships described before.

With $S$, a set of symptoms, and a fuzzy relation $R$ from $S$ to $D$, where $D$ is a set of diseases. And with $P$, a set of patients, and a fuzzy relation $Q$ from $P$ to $S$, and by "max- min composition," we get the fuzzy relation $T=Q \times R$ with the membership function

$$
\begin{array}{r}
\mu_{T}(p, d)=\max _{s \in S}\left(\min \left\{\mu_{Q}(p, s), \mu_{R}(s, d)\right\}\right), \\
s \in S, d \in D, p \in P .
\end{array}
$$

Furthermore, based on the concepts of fuzzy sets (FSs) theory, numerous fuzzy approaches to medical diagnosis have been addressed, and the readers can refer to $[3,5-13]$.

In addition, several investigations in medical diagnosis have addressed these issues based on intuitionistic fuzzy sets (IFSs), such as Shannon et al. [14] developed an intuitionistic fuzzy logic approach for decision making in medicine; De et al. [1] used intuitionistic fuzzy sets to apply to medical diagnosis; Ahn et al. [15] have also presented a medical diagnostic method by applying intuitionistic fuzzy set to perform classification of solution sets and linear regression approach; Innocent and John [16] presented a new method for computing a diagnostic support index which uses vague 
symptom and temporal information in a clinical diagnosis context; Szmidt and Kacprzyk [17] provided a measure method for intuitionistic fuzzy sets and showed its usefulness in medical diagnostic reasoning; $\mathrm{Xu}$ [18] proposed a new method for deriving the correlation coefficients to the interval-valued intuitionistic fuzzy set theory and showed its application in medical diagnosis; Todorova et al. $[19,20]$ provided two algorithms by intuitionistic fuzzy Voronoi diagrams (IFVDs) mechanism to estimate the classification in medicine; Khatibi and Montazer [21] take an intelligent approach towards the bacteria classification problem by using five similarity measures of FSs and IFSs to examine their capabilities in encountering uncertainty in the medical pattern recognition. Currently, Ye [22] also presents a cosine similarity measure and a weighted cosine similarity measure based on the concept of the cosine similarity measure for IFSs.

Furthermore, Vlachos and Sergiadis [23] presented an intuitionistic fuzzy cross-entropy (IFCE) approach to discrimination measures for IFSs and their application in medical diagnosis. Zhang and Jiang [24] have also proposed two kinds of new vague information entropy measures which are vague entropy and vague cross-entropy to solve medical diagnosis problem.

Among the IFCE approach, this paper also provides an improvement to examine their capabilities to cope with uncertainty in the medical pattern recognition.

The remainder of this study is organized as follows. In Section 2, the relative basic concepts including IFSs, fuzzy cross-entropy, and IFCE methods are described. Section 3 presents an improved IFCE approach, illustrates an example for medical diagnosis, and provides some discussions in Section 4. A computer-based interface has developed in Section 5. Finally, a conclusion is drawn in Section 6.

\section{Related Works}

2.1. IFSs Theory. Fuzzy sets theory, proposed by Zadeh [4] in 1965, has shown successful applications in various fields. In this theory, the membership of an element to a fuzzy set is a single value between zero and one. But in reality, it may not always be certain that the degree of nonmembership of an element to a fuzzy set is just equal to 1 minus the degree of membership, that is, there may be some hesitation degree. Thus, as a generalization of fuzzy sets, the concept of IFSs was introduced by Atanassov [25] in 1983. Burillo and Bustince [26] showed that this notion coincides with the notion of vague sets (VSs).

The IFSs is as an extension of fuzzy sets. An IFSs $A$ in a fixed set $E$ is an objective with the expression

$$
A=\left\{\left\langle x, \mu_{A}(x), v_{A}(x)\right\rangle \mid x \in E\right\},
$$

where the functions $\mu_{\mathrm{A}}: \mathrm{E} \rightarrow[0,1]$ and $\nu_{\mathrm{A}}: \mathrm{E} \rightarrow$ $[0,1]$ denote the degree of membership and the degree of nonmembership of the element $x \in E$, respectively. For every $x \in E$,

$$
0 \leq \mu_{A}(x)+v_{A}(x) \leq 1
$$

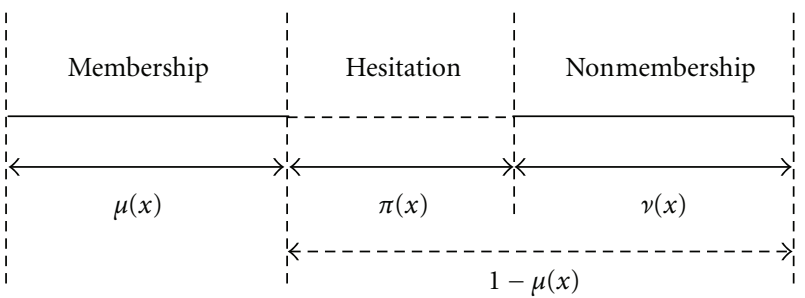

Figure 1: The descriptions of membership, nonmembership and hesitation degree.

When $\mu_{A}(x)+v_{A}(x)=1$, for every $x \in E$, then the IFSs will degenerate to a fuzzy set. Hence, we can consider a fuzzy set with its membership function $\mu_{A}(x)$, having the IFSs expression as

$$
A=\left\{\left\langle x, \mu_{A}(x), 1-\mu_{A}(x)\right\rangle \mid x \in E\right\},
$$

under the condition of $\nu_{A}(x)=1-\mu_{A}(x)$, for every $x \in E$.

For each IFSs $A$ in $E$, "hesitation degree" has consideration. We call the hesitation degree of an element $x \in E$ in $A$ by the following expression:

$$
\pi_{A}(x)=1-\mu_{A}(x)-v_{A}(x),
$$

and we can consider $\pi_{A}(x)$ as a hesitancy degree of $x$ to $A$. From (5), it is evident that

$$
0 \leq \pi_{A}(x) \leq 1, \quad \forall x \in E .
$$

The illustration of these degrees has been exhibited in Figure 1.

Therefore, to describe an intuitionistic fuzzy set completely, we need at least two functions from the triplet [27, 28]: (1) membership function, (2) nonmembership function, and (3) hesitation degree.

2.2. FCE Method. Let us recall the definition of the crossentropy by Kullback [29]. Let $p$ and $q$ be two probability distributions of the discrete random variable $X$. Then, the cross-entropy measure of $p$ from $q$ can be obtained as

$$
I(p, q)=\sum_{x \in X} p(x) \ln \frac{p(x)}{q(x)},
$$

which measures the amount of discrimination of $p$ from $q$. Lin [30] pointed out that (7) is undefined if $q(x)=0$ and $p(x)=0$ for any $x \in E$. To overcome this drawback, He proposed a modified cross-entropy measure described

$$
K(p, q)=\sum_{x \in X} p(x) \ln \frac{2 p(x)}{p(x)+q(x)},
$$

which is well defined and independent of the values of $p(x)$ and $q(x), x \in E$.

Based on (8), Shang and Jiang [31] defined the FCE between two sets $\tilde{A}, \widetilde{B} \in U(X)$, where hereinafter $X$ denotes the finite universe of discourse. Thus, they provided the definition of FCE approach as the following. 
Definition 1 (see [31]). Let $\widetilde{A}$ and $\widetilde{B}$ be two FSs defined on $X$. Then,

$$
E_{\mathrm{FS}}(\widetilde{A}, \widetilde{B})=K\left(\mu_{\widetilde{A}}, \mu_{\widetilde{B}}\right)+K\left(1-\mu_{\widetilde{A}}, 1-\mu_{\widetilde{B}}\right)
$$

is called fuzzy cross-entropy, where $n$ is the cardinality of the finite universe $X$. The equation (9) is the degree of discrimination of $A$ from $B$. However, $E_{\mathrm{FS}}(\widetilde{A}, \widetilde{B})$ is not symmetric with respect to its arguments. Thus, Shang and Jiang [31] also proposed a symmetric discrimination information measure based on $E_{\mathrm{FS}}(\widetilde{A}, \widetilde{B})$, given by

$$
D_{\mathrm{FS}}(\widetilde{A}, \widetilde{B})=E_{\mathrm{FS}}(\widetilde{A}, \widetilde{B})+E_{\mathrm{FS}}(\widetilde{B}, \widetilde{A}) .
$$

Moreover, they showed that $D_{\mathrm{FS}}(\widetilde{A}, \widetilde{B}) \geq 0$ and $D_{\mathrm{FS}}(\widetilde{\mathrm{A}}, \widetilde{\mathrm{B}})=0$ if and only if $\widetilde{\mathrm{A}}=\widetilde{\mathrm{B}}$.

2.3. IFCE Method. Let us consider two sets $A, B \in U(X)$. In order to derive a cross-entropy measure for IFSs, we have to exploit the information carried by both the membership and the nonmembership function. In an analogous manner to Bhandari and Pal [32], we consider the following quantity:

$$
I^{\mu}\left(A, B ; x_{i}\right)=\ln \frac{\mu_{A}\left(x_{i}\right)}{\mu_{B}\left(x_{i}\right)},
$$

as the amount of information for discrimination of $\mu_{A}\left(x_{i}\right)$ from $\mu_{B}\left(x_{i}\right)$. Therefore, the expected information for discrimination of $A$ against $B$, based solely on the membership function, is given by

$$
I^{\mu}(A, B)=\sum_{i=1}^{n} \mu_{A}\left(x_{i}\right) \ln \frac{\mu_{A}\left(x_{i}\right)}{\mu_{B}\left(x_{i}\right)} .
$$

Similarly, considering the nonmembership function that we have,

$$
I^{\nu}(A, B)=\sum_{i=1}^{n} v_{A}\left(x_{i}\right) \ln \frac{\nu_{A}\left(x_{i}\right)}{\nu_{B}\left(x_{i}\right)} .
$$

Hence, the information for discrimination in favor of $A$ against $B$ is obtained as the sum of the quantities $I^{\mu}(A, B)$ and $I^{\nu}(A, B)$, that is,

$$
I_{\mathrm{IFS}}^{\prime \prime}(A, B)=I^{u}(A, B)+I^{v}(A, B) .
$$

In order to overcome the drawback of (14) being undefined if $\mu_{B}\left(x_{i}\right)=0$ or $v_{B}\left(x_{i}\right)=0$ for any $x_{i} \in X$, a modified version of (14) is introduced based on (8) and is given by

$$
I_{\mathrm{IFS}}(A, B)=K\left(\mu_{A}, \mu_{B}\right)+K\left(\nu_{A}, \nu_{B}\right) .
$$

According to Shannon's inequality, Lin [30] pointed out that one can easily prove that $I_{\mathrm{IFS}}(A, B) \geq 0$ and $I_{\mathrm{IFS}}(A, B)=0$ if and only if $A=B$. A similar measure for FSs, denoted by $\mathrm{I}_{\mathrm{FS}}(\widetilde{\mathrm{A}}, \widetilde{\mathrm{B}})$, was defined by Bhandari and Pal [32]. For FSs in general $I_{\mathrm{FS}}(\widetilde{A}, \widetilde{B}) \neq I_{\mathrm{FS}}\left(\widetilde{A}^{c}, \widetilde{B}^{c}\right)$. However, for IFSs, the following equality holds:

$$
I_{\mathrm{IFS}}(A, B)=I_{\mathrm{IFS}}\left(A^{c}, B^{c}\right),
$$

where $A^{c}$ and $B^{c}$ are the complementary sets of $A$ and $B$, respectively. Therefore, in analogy with (9), we proceed to the following definition of the intuitionistic fuzzy cross-entropy.

Definition 2. For two sets $A, B \in U(X), I_{\mathrm{IFS}}(A, B)$ is the intuitionistic fuzzy cross-entropy between $A$ and $B$. $I_{\mathrm{IFS}}(A, B)$ can also be called discrimination information for IFSs.

However, one can observe that $I_{\mathrm{IFS}}(A, B)$ is not symmetric with respect to its arguments. Therefore, a symmetric measure is defined as follows.

Definition 3. For two sets $A, B \in U(X)$,

$$
D_{\mathrm{IFS}}(A, B)=I_{\mathrm{IFS}}(A, B)+I_{\mathrm{IFS}}(B, A)
$$

is called a symmetric discrimination information measure for IFSs.

It can easily be verified that $D_{\mathrm{IFS}}(A, B) \geq 0$ and $D_{\mathrm{IFS}}(A, B)=0$ if and only if $A=B$. Moreover, $I_{\mathrm{IFS}}(A, B)$ and $D_{\mathrm{IFS}}(A, B)$ degenerate to their fuzzy counterparts when $A$ and $B$ are FSs.

\section{An Improved IFCE Approach}

It is easy to see that cross-entropy proposed by Vlachos and Sergiadis [23] only involve the first two parameters, that is, the membership degree and nonmembership degree of IFSs. Furthermore, it does not take the third parameter (hesitation) into account in the above derivation and discrimination measure of IFSs.

Furthermore, Szmidt and Kacprzyk [33] have showed that omitting one of the three parameters may lead to incorrect results, and thus all the three parameters should be taken into account when calculating association or distance between two IFSs.

Motivated by the idea of Szmidt and Kacprzyk [33], we improve the feasibility of original IFCE by adding the third parameter (hesitation degree) consideration. Therefore, a modified formulation is improved to reach better discrimination measure of IFCE approach.

Following (12) and (13), we may keep considering hesitation function for discrimination measure similarly. Thus, we can obtain

$$
I^{\pi}(A, B)=\sum_{i=1}^{n} \pi_{A}\left(x_{i}\right) \ln \frac{\pi_{A}\left(x_{i}\right)}{\pi_{B}\left(x_{i}\right)} .
$$

Next, the information for discrimination in favor of $A$ against $B$ can be calculated as the sum of the quantities $I^{\mu}(A, B)$, $I^{v}(A, B)$, and $I^{\pi}(A, B)$. The equation (14) can be revised as

$$
I_{\mathrm{IFS}}^{\prime}(A, B)=I^{u}(A, B)+I^{\nu}(A, B)+I^{\pi}(A, B) .
$$

To avoid the drawback of (19) being undefined if $\mu_{B}\left(x_{i}\right)=0$, $\nu_{B}\left(x_{i}\right)=0$ or $\pi_{B}\left(x_{i}\right)=0$ for any $x_{i} \in X$, an improved version of (19) is introduced based on (15) and is given as

$$
I_{\mathrm{IFS}}(A, B)=K\left(\mu_{A}, \mu_{B}\right)+K\left(\nu_{A}, \nu_{B}\right)+K\left(\pi_{A}, \pi_{B}\right) .
$$


TABLE 1: Symptoms characteristic for the diagnoses considered.

\begin{tabular}{lcccrr}
\hline & Viral fever & Malaria & Typhoid & \multicolumn{1}{c}{$\begin{array}{c}\text { Stomach } \\
\text { problem }\end{array}$} & $\begin{array}{c}\text { Chest } \\
\text { problem }\end{array}$ \\
\hline Temperature & $(0.4,0.0,0.6)$ & $(0.7,0.0,0.3)$ & $(0.3,0.3,0.4)$ & $(0.1,0.7,0.2)$ & $(0.1,0.8,0.1)$ \\
Headache & $(0.3,0.5,0.2)$ & $(0.2,0.6,0.2)$ & $(0.6,0.1,0.3)$ & $(0.2,0.4,0.4)$ & $(0.0,0.8,0.2)$ \\
Stomach pain & $(0.1,0.7,0.2)$ & $(0.0,0.9,0.1)$ & $(0.2,0.7,0.1)$ & $(0.8,0.0,0.2)$ & $(0.2,0.8,0.0)$ \\
Cough & $(0.4,0.3,0.3)$ & $(0.7,0.0,0.3)$ & $(0.2,0.6,0.2)$ & $(0.2,0.7,0.1)$ & $(0.2,0.8,0.0)$ \\
Chest pain & $(0.1,0.7,0.2)$ & $(0.1,0.8,0.1)$ & $(0.1,0.9,0.0)$ & $(0.2,0.7,0.1)$ & $(0.8,0.1,0.1)$ \\
\hline
\end{tabular}

TABLE 2: Symptoms characteristic for the patients considered.

\begin{tabular}{lccccc}
\hline & Temperature & Headache & Stomach pain & Cough & Chest pain \\
\hline Al & $(0.8,0.1,0.1)$ & $(0.6,0.1,0.3)$ & $(0.2,0.8,0.0)$ & $(0.6,0.1,0.3)$ & $(0.1,0.6,0.3)$ \\
Bob & $(0.0,0.8,0.2)$ & $(0.4,0.4,0.2)$ & $(0.6,0.1,0.3)$ & $(0.1,0.7,0.2)$ & $(0.1,0.8,0.1)$ \\
Joe & $(0.8,0.1,0.1)$ & $(0.8,0.1,0.1)$ & $(0.0,0.6,0.4)$ & $(0.2,0.7,0.1)$ & $(0.0,0.5,0.5)$ \\
Ted & $(0.6,0.1,0.3)$ & $(0.5,0.4,0.1)$ & $(0.3,0.4,0.3)$ & $(0.7,0.2,0.1)$ & $(0.3,0.4,0.3)$ \\
\hline
\end{tabular}

One can also prove that $I_{\mathrm{IFS}}(A, B) \geq 0$ and $I_{\mathrm{IFS}}(A, B)=0$ if and only if $A=B$, according to Shannon's inequality. A similar measure for IFSs, the following equality also holds:

$$
I_{\mathrm{IFS}}(A, B)=I_{\mathrm{IFS}}\left(A^{c}, B^{c}\right),
$$

where $A^{c}$ and $B^{c}$ are the complementary sets of $A$ and $B$, respectively. Therefore, in analogy with (20), we can also proceed to the symmetric discrimination definition of the proposed intuitionistic fuzzy cross-entropy

$$
D_{\mathrm{IFS}}(A, B)=I_{\mathrm{IFS}}(A, B)+I_{\mathrm{IFS}}(B, A) .
$$

\section{Medical Diagnosis Example and Discussions}

4.1. Illustrative Example for Medical Diagnosis. We will illustrate an application using the improved IFCE approach for medical pattern recognition problem.

Let us consider the same example in De et al. [1] and Szmidt and Kacprzyk $[34,35]$. They consist of a set of patients $P=\mathrm{Al}$, Bob, Joe, Ted, a set of diagnoses $D=$ viral fever, malaria, typhoid, stomach problem, chest pain, and a set of symptoms $S$ = temperature, headache, stomach pain, cough, chest pain. Table 1 presents the characteristic symptoms for the diagnoses considered. The symptoms for each patient are given in Table 2. Each element of the tables is given in the form of a pair of numbers corresponding to the membership, nonmembership, and hesitation values, respectively, for example, the temperature for viral fever is described by $(\mu, \nu, \pi)=(0.4,0,0.6)$ in Table 1 .

In order to find a proper diagnosis, we calculate for each patient $p_{i} \in P$, where $i \in\{1, \ldots, 4\}$, the symmetric discrimination information measure for IFSs $D_{\mathrm{IFS}}\left(s\left(p_{i}\right), d_{k}\right)$ between patient symptoms and the set of symptoms that are characteristic for each diagnosis $d_{k} \in D$, with $k \in\{1, \ldots, 5\}$. Based on (20) and (22), we assign to the ith patient the diagnosis whose symptoms have the lowest symmetric discrimination information measure from patient's symptoms. The diagnosed results for the considered patients are given in Table 3. We assign to the ith patient the diagnosis whose symptoms have the lowest symmetric discrimination information measure from patient's symptoms. The diagnosed results for the considered patients are given in Table 3 .

According to Table 3, we know that Al, Joe, and Ted maybe suffer viral fever whereas Bob faces a stomach pain problem.

4.2. Discussions. Through this study, some issues can be raised and depicted as follows.

(1) From the practical case of medical diagnosis study, the proposed method can provide a useful way to help doctors perform preliminary diagnosis. The proposed method differs from previous methods for medical diagnosis decision making due to the fact that the proposed method considers the degree of hesitation compared to other existing approaches. In the future, the proposed method may be a merit for the preliminary diagnosed models to solve the medical diagnosis problem using IFSs.

(2) Sometimes, the difference between two adjacent ranking scores is very close. Observing Table 3 , the suffered outcomes of AL, the scores of viral fever and malaria are close. Joe suffered from viral fever and typhoid, of which the scores are close. If it appears in the first and second rank, doctors require making an advanced diagnosis with their expert knowledge to evaluate the values of two IFSs because the doctor process allows little deficiency caused by the practical case study among uncertain environment and usage to achieve the optimal judgment. Therefore, the purpose of advanced medical diagnosis is to reduce the risk which may occur in medical diagnosis.

\section{A Computer-Based Interface}

As more and more decisions in real organizational settings are made, applying IFSs into medical diagnosis analysis to deal with imprecision, uncertainty, and fuzziness in decision making may become a popular research topic in 
TABLE 3: The diagnosed results by the improved IFCE approach.

\begin{tabular}{lccccc}
\hline & Viral fever & Malaria & Typhoid & $\begin{array}{c}\text { Stomach } \\
\text { problem }\end{array}$ & $\begin{array}{c}\text { Chest } \\
\text { problem }\end{array}$ \\
\hline Al & $\mathbf{0 . 7 8 7 3}$ & 0.7885 & 0.8902 & 2.1573 & 2.5384 \\
Bob & 1.6195 & 2.6886 & 0.9896 & $\mathbf{0 . 2 8 8 6}$ & 1.8327 \\
Joe & $\mathbf{1 . 0 4 4 1}$ & 1.5938 & 1.0668 & 2.2708 & 2.8497 \\
Ted & $\mathbf{0 . 4 9 9 7}$ & 0.8992 & 0.9952 & 1.4273 & 1.9536 \\
\hline
\end{tabular}

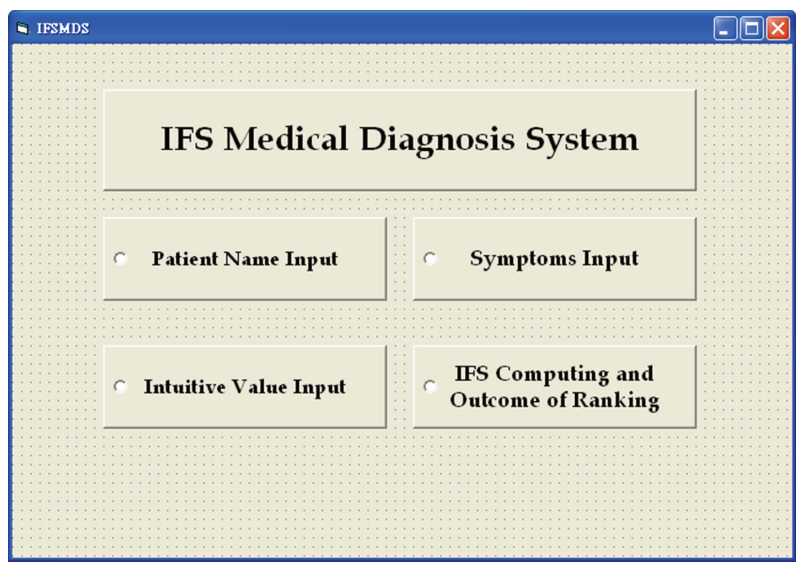

FIgURE 2: The functional interface of IFSMDS.

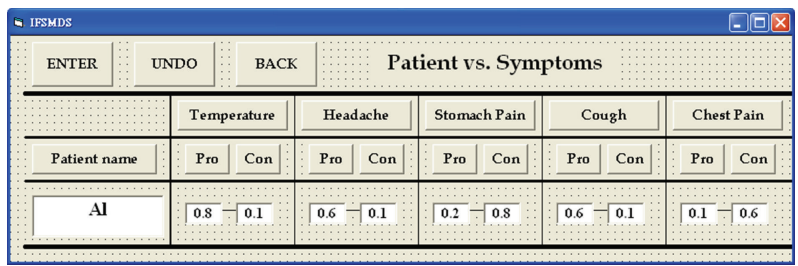

FIGURE 3: Input intuitionistic diagnosed value on each symptom for patient.

the current uncertain environment. The application of IFSs in supporting doctors can provide a useful way to help the decision analyzer make his/her decisions efficiently.

In this paper, in order to make computing and ranking the results much easier and to increase the recruiting productivity, we have developed an information system called intuitionistic fuzzy sets medical diagnosis system (IFSMDS) as shown in Figure 2. This prototype system is developed by Visual Basic 6 and ACCESS on a $N$-tier client server architecture. In IFCEDSS, doctors need to key in the patient's name and symptoms data, respectively. The intuitive values of each patient on each relation between symptoms and diseases have showed, as illustrated in Figure 3. The system can calculate the assessment value of each patient on each symptom. The diagnosed results are shown as Figure 4. If ranking score is the lowest, then the patient is more likely to suffer from the disease.

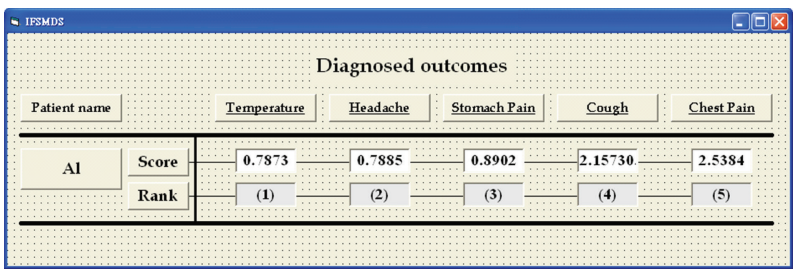

FIgURE 4: The outcomes and ranking of medical diagnosis.

\section{Concluding and Remarks}

In this paper, an improved IFCE approach is presented based on IFSs. The idea of improvement is to add the hesitation degree and reveal an intuitive and mathematical connection between the notions of entropy for IFSs in terms of fuzziness and intuitionism based on this entropy measure. Illustrative examples have demonstrated the usefulness of the proposed discrimination information measure for medical diagnosis. In addition, in order to prevent misjudgment, we have suggested that when the diagnosed results between the diseases are very close, an advanced diagnosis is necessary to avoid some error risks of the medical diagnosis.

\section{Acknowledgments}

The author would like to express appreciation to the anonymous reviewers for their very helpful comments on improving this paper. This research is partially supported by the National Science Council of the Republic of China, Taiwan with Grant no. NSC 100-2410-H-606-002-MY2.

\section{References}

[1] S. K. De, R. Biswas, and A. R. Roy, "An application of intuitionistic fuzzy sets in medical diagnosis," Fuzzy Sets and Systems, vol. 117, no. 2, pp. 209-213, 2001.

[2] E. Straszecka, "Combining uncertainty and imprecision in models of medical diagnosis," Information Sciences, vol. 176, no. 20, pp. 3026-3059, 2006.

[3] E. Sanchez, "Medical diagnosis and composite fuzzy relations," in Advances in Fuzzy Set Theory and Applications, M. M. Gupta, R. K. Ragade, and R. R. Yager, Eds., North-Holland, Amsterdam, The Netherlands, 1979.

[4] L. A. Zadeh, "Fuzzy sets," Information and Control, vol. 8, no. 3, pp. 338-353, 1965.

[5] F. Steimann and K. P. Adlassnig, "Fuzzy Medical Diagnosis," http://citeseerx.ist.psu.edu/viewdoc/summary?doi=10.1.1 .47.9705. 
[6] F. F. Yao and J. S. Yao, "Fuzzy decision making for medical diagnosis based on fuzzy number and compositional rule of inference," Fuzzy Sets and Systems, vol. 120, no. 3, pp. 351-366, 2001.

[7] J. Palma, J. M. Juarez, M. Campos, and R. Marin, "Fuzzy theory approach for temporal model-based diagnosis: an application to medical domains," Artificial Intelligence in Medicine, vol. 38, no. 2, pp. 197-218, 2006.

[8] R. Seising, "From vagueness in medical thought to the foundations of fuzzy reasoning in medical diagnosis," Artificial Intelligence in Medicine, vol. 38, no. 3, pp. 237-256, 2006.

[9] A. Quteishat and C. P. Lim, "Application of the fuzzy minmax neural networks to medical diagnosis," Lecture Notes in Computer Science, vol. 5179, part 3, pp. 548-555, 2008.

[10] I. Gadaras and L. Mikhailov, "An interpretable fuzzy rulebased classification methodology for medical diagnosis," Artificial Intelligence in Medicine, vol. 47, no. 1, pp. 25-41, 2009.

[11] C. M. Own, "Switching between type-2 fuzzy sets and intuitionistic fuzzy sets: an application in medical diagnosis," Applied Intelligence, vol. 31, no. 3, pp. 283-291, 2009.

[12] S. Badaloni and M. Falda, "Temporal-based medical diagnoses using a fuzzy temporal reasoning system," Journal of Intelligent Manufacturing, vol. 21, no. 1, pp. 145-153, 2010.

[13] S. Lekkas and L. Mikhailov, "Evolving fuzzy medical diagnosis of Pima Indians diabetes and of dermatological diseases," Artificial Intelligence in Medicine, vol. 50, no. 2, pp. 117-126, 2010.

[14] A. Shannon, S. Kim, Y. Kim, J. Sorsich, K. Atanassov, and P. Georgiev, "A possibility for implementation of elements of the intuitionistic fuzzy logic in decision making in medicine," in Proceedings of the First International Conference on Intuitionistic Fuzzy Sets, J. Kacprzyk and K. Atanassov, Eds., vol. 3 of Notes on intuitionistic fuzzy sets, no. 4, pp. 40-43, 1997.

[15] J. Y. Ahn, Y. H. Kim, and S. K. Kim, "A fuzzy differential diagnosis of headache applying linear regression method and fuzzy classification," IEICE Transactions on Information and Systems, vol. E86-D, no. 12, pp. 2790-2793, 2003.

[16] P. R. Innocent and R. I. John, "Computer aided fuzzy medical diagnosis," Information Sciences, vol. 162, no. 2, pp. 81-104, 2004.

[17] E. Szmidt and J. Kacprzyk, "A similarity measure for intuitionistic fuzzy sets and its application in supporting medical diagnostic reasoning," in Proceedings of the Lecture Notes in Computer Science (ICAISC '04), L. Rutkowski, J. H. Siekmann, R. Tadeusiewicz, and L. A. Zadeh, Eds., vol. 3070, pp. 388-393, Springer, Berlin, Germany, 2004.

[18] Z. S. Xu, "On correlation measures of intuitionistic fuzzy sets," Lecture Notes in Computer Science, vol. 4224, pp. 16-24, 2006.

[19] L. Todorova, K. Atanassov, S. Hadjitodorov, and P. Vassilev, "On an intuitionistic fuzzy approach for decision making in medicine: part 1," International Journal Bioautomation, vol. 6, pp. 92-101, 2007.

[20] L. Todorova, K. Atanassov, S. Hadjitodorov, and P. Vassilev, "On an intuitionistic fuzzy approach for decision making in medicine: part 2," International Journal Bioautomation, vol. 7, pp. 64-69, 2007.

[21] V. Khatibi and G. A. Montazer, "Intuitionistic fuzzy set vs. fuzzy set application in medical pattern recognition," Artificial Intelligence in Medicine, vol. 47, no. 1, pp. 43-52, 2009.

[22] J. Ye, "Cosine similarity measures for intuitionistic fuzzy sets and their applications," Mathematical and Computer Modelling, vol. 53, no. 1-2, pp. 91-97, 2011.
[23] I. K. Vlachos and G. D. Sergiadis, "Intuitionistic fuzzy information-applications to pattern recognition," Pattern Recognition Letters, vol. 28, no. 2, pp. 197-206, 2007.

[24] Q. S. Zhang and S. Y. Jiang, "A note on information entropy measures for vague sets and its applications," Information Sciences, vol. 178, no. 21, pp. 4184-4191, 2008.

[25] K. Atanassov, "Intuitionistic fuzzy sets," VII ITKR Session, Sofia (Deposed in Central Library Of The Bulgarian Academy Of Sciences, 1697/84), 1983, (in Bulgarian).

[26] P. Burillo and H. Bustince, "Entropy on intuitionistic fuzzy sets and on interval-valued fuzzy sets," Fuzzy Sets and Systems, vol. 78, no. 3, pp. 305-316, 1996.

[27] K. T. Atanassov, "More on intuitionistic fuzzy sets," Fuzzy Sets and Systems, vol. 33, no. 1, pp. 37-45, 1989.

[28] K. Atanassov, Intuitionistic Fuzzy Sets: Theory and Applications, Physica, Heidelberg, Germany, 1999.

[29] S. Kullback, Information Theory and Statistics, Dover, New York, NY, USA, 1968.

[30] J. Lin, "Divergence measures based on the Shannon entropy," IEEE Transactions on Information Theory, vol. 37, no. 1, pp. 145-151, 1991.

[31] X. G. Shang and W. S. Jiang, "A note on fuzzy information measures," Pattern Recognition Letters, vol. 18, no. 5, pp. 425432, 1997.

[32] D. Bhandari and N. R. Pal, "Some new information measures for fuzzy sets," Information Sciences, vol. 67, no. 3, pp. 209-228, 1993.

[33] E. Szmidt and J. Kacprzyk, "Distances between intuitionistic fuzzy sets," Fuzzy Sets and Systems, vol. 114, no. 3, pp. 505$518,2000$.

[34] E. Szmidt and J. Kacprzyk, "Intuitionistic fuzzy sets in intelligent data analysis for medical diagnosis," in Proceedings of the Computational Science (ICCS '01), V. N. Alexandrov, J. J. Dongarra, B. A. Juliano, R. S. Renner, and C. J. K. Tan, Eds., vol. 2074, pp. 263-271, Springer, Berlin, Germany, 2001.

[35] E. Szmidt and J. Kacprzyk, "Intuitionistic fuzzy sets in some medical applications," in Proceedings of the 7th Fuzzy Days Dortmund, B. Reusch, Ed., vol. 2206 of Computational intelligence: theory and applications, pp. 148-151, Springer, Berlin, Germany, 2001. 

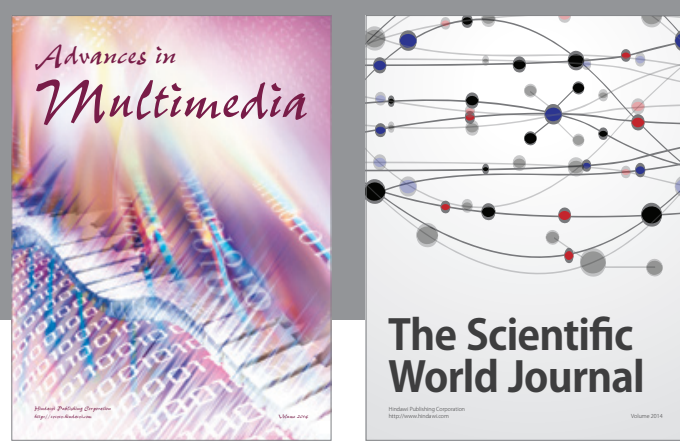

The Scientific World Journal
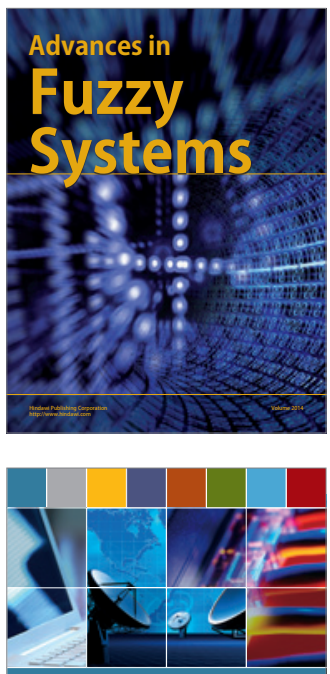

Computer Networks and Communications
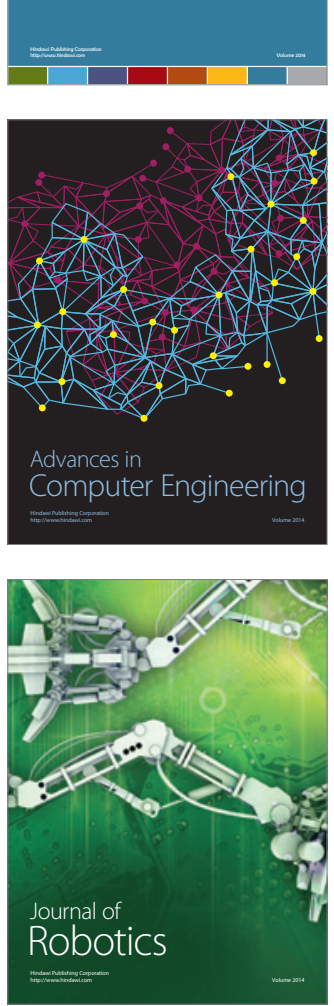
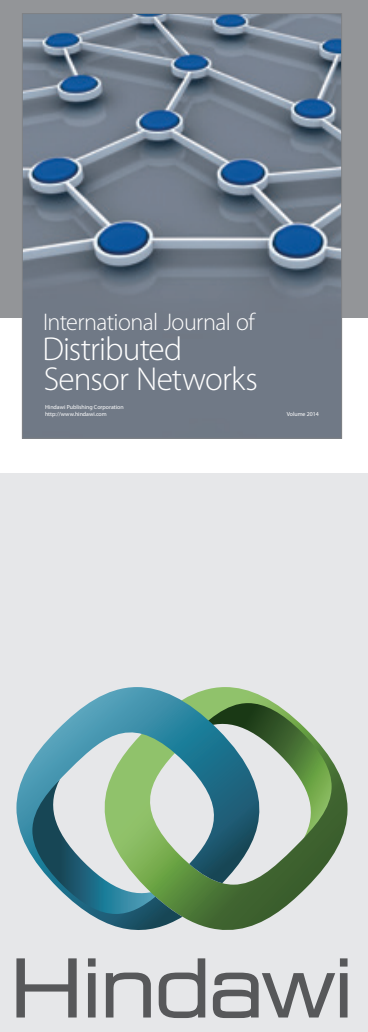

Submit your manuscripts at

http://www.hindawi.com
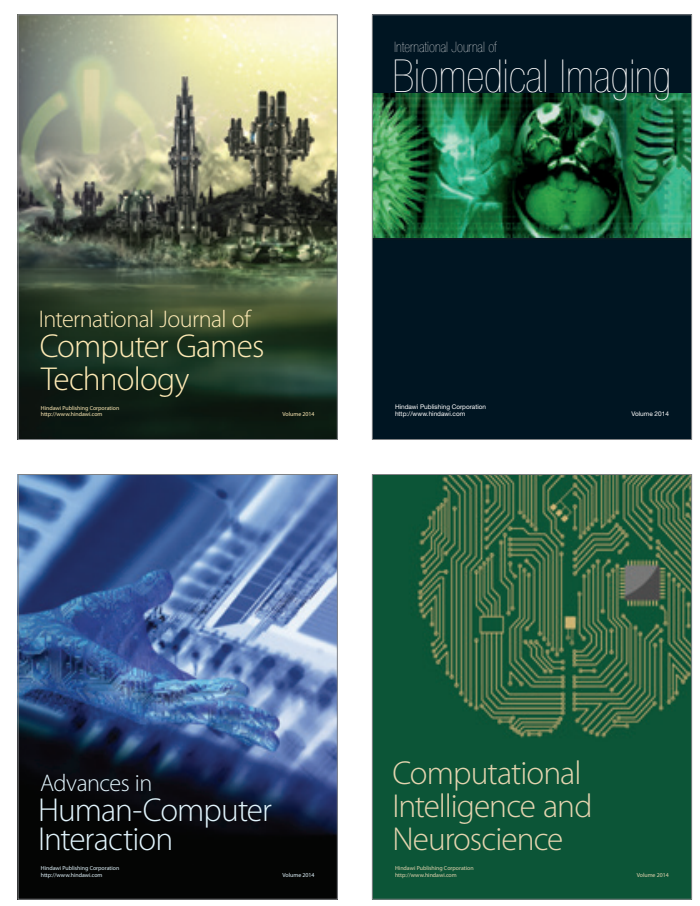
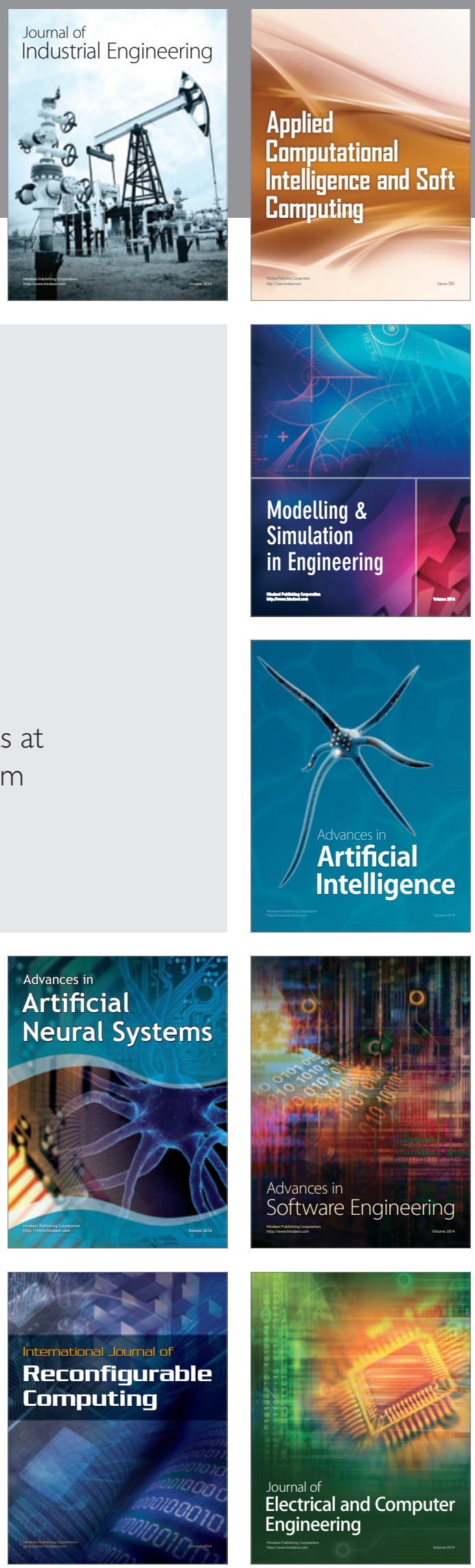